\title{
INMIGRACIÓN E INTEGRACIÓN
}

\author{
Fausto Miguélez \\ (Universidad Autónoma de Barcelona)
}

Recientemente el tema de las migraciones ha vuelto a tener importancia entre los sociólogos desde diversas perspectivas, ${ }^{1}$ con razón, a mi entender, puesto que se trata de una consecuencia de la vuelta del tema al escenario político y económico.

En efecto, el debate en torno al estado de las autonomías y, sobre todo, sus repercusiones en el ámbito del gobierno autonómico, de la cultura autóctona, de la lengua, de la organización de partidos y sindicatos de ámbito de nacionalidad nos ha puesto sobre la mesa, de manera práctica, que un porcentaje muy elevado de catalanes y vascos provienen de otras tierras de España, que multitud de gallegos, andaluces, castellanos y un largo etcétera están, definitivamente, asentados en zonas diversas a las de su origen. ¿Definitivamente? He aquí un segundo aspecto, junto con la migración propiamente tal, que la crisis económica ha puesto asimismo de actualidad. El retorno de emigrantes establecidos hace años en Europa es un hecho que se constata en las estadísticas - en manera, se entiende, más determinante que en años anteriores-y del que están apareciendo

1. Véanse, entre otros:

E. Pinilla de las Heras: Estudios sobre cambio social y estructuras sociales en $\mathrm{Ca}$ taluña, CIS, Madrid, 1979.

- Immigració i mobilitat social a Catalunya, ICESB, Barcelona, 1973-78, 5 vol.

J. Cardelús y A. Pascual: Movimientos migratorios y organización social, Península, Barcelona, 1980. 
estudios y publicaciones. ${ }^{2}$ También hay indicios de retornos interiores; la misma provincia de Barcelona tiene saldo migratorio negativo desde hace dos años. Sin embargo, el retorno interior difícilmente va a llegar a ser un fenómeno sociológicamente muy significativo. Son muchos los lazos que atan a la sociedad receptora: la casa en propiedad a menudo, los hijos, su educación y sus expectativas y la esperanza de que la crisis toque finalmente fondo, mientras que las zonas de origen no aparecen, sino todo lo contrario, como pródigas de puestos de trabajo.

El problema de fondo de quienes han emigrado y no piensan retornar es el de la integración, es decir, la inserción en la sociedad receptora a la que han decidido vincular definitivamente su proyecto de vida. Sobre este tema, y sobre el libro de Carlota Solé, La integración socio-cultural de los inmigrantes en Cataluña, CIS, Madrid, 1981, versará esta nota.

La autora del estudio constata que sólo un $9 \%$ de los inrnigrantes a Cataluña admiten que volverían a su tierra, puesto que piensan que allí se encontrarían mejor personalmente. Por tanto, la integración y el no retorno se plantea, principalmente, para aquellos que vinieron acuciados por la necesidad y que difícilmente pueden pensar en una salida diferente -excepto otra emigración en el mismo sentido- a lo que es esta nueva vida. Este tipo de emigración plantea el tema de la integración, sobre todo, en relación con las clases trabajadoras. Pero, al mismo tiempo, la no voluntariedad de la condición emigrante hace extraordinariamente complejo el tema de la integración.

\section{EL AMPLIO ORIGEN MIGRATORIO DE LA CLASE OBRERA CATALANA}

Las migraciones interiores han constituido uno de los más importantes factores de cambio social en la España y Cataluña actuales. Pero este cambio en Cataluña y en otras regiones receptoras ha tenido un signo muy diverso del adoptado en las zonas emisoras, al menos por: lo que se refiere a la estructura social; ciertos grupos sociales (pequeño campesinado, braceros agrícolas, pequeña y mediana burguesía rural han perdido peso

2. J. Cazorla Pérez: Emigración y retorno. Una perspectiva europea, Instituto Español de Emigración, Madrid, 1981.

J. Castillo Castillo: La emigración española en la encrucijada. Análisis empírico de la emigración de retorno, CIS, Madrid, 1980.

A. Pascual: El retorno de migrantes españoles de Europa. Tesis doctoral presentada en la Universidad Autónoma de Barcelona, marzo 1982. 
cuantitativa y políticamente), otros (la clase obrera, los empleados y técnicos) no sólo han aumentado, sino que se han transformado interiormente en manera notable a través del fenómeno migratorio. Cambios en pautas de conducta, en comportamientos culturales y políticos, en opiniones y actitudes tienen ahí uno de los factores explicativos.

Pero centrémonos sobre Cataluña y las transformaciones en las clases trabajadoras. De 1941 a 1950 inmigran a Cataluña 256.000 personas, casi 440.000 entre 1951 y 1960 , más de 700.000 en la década siguiente y 205.000 entre 1971 y 1975 . Posteriormente los saldos migratorios favorables a Cataluña son poco significativos. Hoy el $40 \%$ de la población catalana es inmigrante. De ellos, la inmensa mayoría son trabajadores $y$, en particular, se concentran en la clase obrera manual más descualificada de la industria (construcción, metal) y de los servicios (hostelería, limpieza, servicios personales). Un estudio realizado sobre los datos del censo de $1970^{3}$ pone de maniffesto que el $59,59 \%$ de los inmigrantes activos son obreros cualificados o sin cualificar, el 9,35\% trabajadores de los servicios y el $12,49 \%$ personal administrativo y técnico bajo de empresas. Casi el $80 \%$ de los inmigrantes entre estos tres sectores.

Descualificación, manualidad, bajo nivel educativo son características que hoy tiene la clase obrera catalana y que influyen en los procesos de integración.

\section{INTEGRACIÓN CON DISCRIMINACIÓN}

Carlota Solé aborda, pues, en su libro una cuestión fundamental: la integración de los inmigrantes en Cataluña. Trata el tema desde una perspectiva socio-políticamente relevante, es decir, la de la inserción social de los inmigrantes en una sociedad receptora con características históricas, linguísticas, de estructura social, tradiciones y formas políticas, cultura y modos de vida muy peculiares.

El tema de la integración de los inmigrantes ha sido, tradicionalmente, en Cataluña, objeto de debate entre políticos, estudiosos y escritores ${ }^{4}$ a

3. Inventica: Migraciones interiores en España, Dirección General de Planificación Social, Madrid, 1975, tomo I, vol. 2.

4. A. M. Badia: Llengua i cultura als Països Catalans, Edicions 62, Barcelona, 1966.

J. Pujol: La immigració, problema i esperança de Catalunya, Nova Terra, Barcelona, 1966.

F. Candel: Els altres catalans, Edicions 62, Barcelona, 1964.

A. Jutglar y otros: La immigracion a Cataluña, Edima, Barcelona, 1968. 
lo largo de las últimas dos décadas, pero la polémica ha adquirido últimamente tonalidades nuevas debido a su importancia política.

En algunas obras anteriores la idea de la integración tiende a coincidir con la asimilación idiomática o la imitación de rasgos culturales (Badia) o bien con la adaptación pasiva a una estructura social, política y cultural existente ( $\mathrm{J}$. Pujol); se trata, en ambos casos, de una concepción de la sociedad catalana como de algo mecánico y acabado que se fuera transmitiendo de generación en generación.

La obra que comentamos parte de una concepción muy distinta de la integración socio-cultural, entendida como «la interpenetración de los miembros y elementos culturales de dos poblaciones en una única y nueva estructura social y cultura». Históricamente ha sido siempre así y también lo es ahora, según este modo de entender las cosas; la interpenetración entre población autóctona e inmigrante ha influido y seguirá influyendo en el surgir de una estructura de clases nueva, de una cultura y aun de una idiosincrasia diferentes. Este proceso, en que los elementos existentes se entrecruzan con los que llegan, es algo dinámico, dialéctico, alternante y tiene poco que ver con la pasividad y la colonización. Éste es el proceso que se investiga en la obra que comentamos y su principal mérito es haber entrado, por primera vez, a analizar las diversas fases, la estructura interna, las contradicciones internas de la integración.

Pero obviamente, la integración así entendida responde a ciertos presupuestos sociales y políticos que no son los únicos. Hay otras posibles opciones como la no integración que, políticamente, ha sido instrumentalizada en la historia de Cataluña por el fenómeno del lerrouxismo y que algunos parecen dispuestos a resucitar hoy, o el asimilacionismo de quienes se creen superiores, o con derecho de herencia, y exigen asentimiento y subordinación política.

La integración, entendida como coprotagonismo, es, de todos modos, un proceso global y complejo que, en el presente estudio, se desglosa en cuatro subprocesos:

- Integración al trabajo urbano-industrial.

- Adaptación al tipo de vida urbano.

- Aceptación progresiva, libre y voluntaria, de las instituciones sociales y políticas catalanas, en pie de igualdad.

- Adopción voluntaria, progresiva y libre de las normas, costumbres y valores de la nueva sociedad — que son móviles y dinámicas$\mathrm{y}$ de su misma lengua. 
La procedencia rural, y a menudo agrícola, de la mayoría de los inmigrantes, plantea el problema de su integración en una nueva estructura ocupacional que, obviamente, va a ser la base de su modo de vida en la ciudad y de su inserción en la nueva estructura social. Que la inmensa mayoría de los inmigrantes se conviertan en asalariados es un supuesto que no nos dice lo suficiente sobre cómo se realiza ese fenómeno, en qué relación con los nacidos en Cataluña, con qué consecuencias. Cuestiones fundamentales para entender el status social y, más allá de éste, la conciencia frente a la realidad de la nueva sociedad, de los venidos de fuera.

Ya Esteban Pinilla de las Heras en un minucioso estudio sobre inmigración y estructura ocupaciona ${ }^{5}$ se había planteado el tema que nos ocupa desde el punto de vista de la movilidad laboral. Aunque con técnicas diferentes y una categorización no coincidente, ambos autores - Pinilla y C. Solé - abordan, a una distancia de diez años, un problema crucial para la integración. Las conclusiones del estudio de Pinilla de las Heras, que, de algún modo, corroboraban las de Maluquer Sostres una década antes, ${ }^{6}$ venían a señalar que, a más baja categoría laboral, mayor presencia de inmigrantes, y viceversa. Pero dentro de la misma categoría los nativos ocuparían los niveles de mayor responsabilidad y decisión (en las categorías altas) o de mayor cualificación (en las categotías bajas).

La educación parecía ser el factor diferenciador básico, como se desprende del análisis de una submuestra de directivos y técnicos donde los inmigrantes jóvenes, con mayor formación, estarían mejor colocados que los adultos. Pero quizá no sería el único factor, dado que el análisis de las diversas franjas gerenciales y técnicas en relación con la edad, y para el subconjunto de jóvenes, vuelve a señalar una mejor posición para los nativos.

La investigación de Pinilla de las Heras avanza algunas hipótesis para explicar ese hecho, como son: el tipo de estudios realizados, el mejor conocimiento del sistema productivo por los autóctonos, el tamaño de la empresa (en las grandes parece haber mayor igualdad de oportunidades), etc.

$\mathrm{El}$ estudio de C. Solé vuelve a registrar, con gran nitidez, que la población inmigrante ocupa los estratos inferiores de la estructura ocupacional, pero permite entrever un elemento nuevo al constatar esa peor posición de los nacidos en el resto de España frente no sólo a los nacidos en Cataluña, sino en toda el área lingüística catalana. ¿'Tiene la lengua algún peso diferenciador? Volveremos inmediatamente sobre este tema.

5. E. Pinilla de las Heras: Immigració i mobilitat social a Catalunya, op. cit.

6. J. Maluquer i Sostres: L'assimilation des immigrés en Catalogne, Librairie Droz, Ginebra, 1963. 
Comparando el actual con el anterior empleo, el estudio La integración socio-cultural... constata una cierta movilidad ocupacional, es decir, un aumento de la proporción de inmigrantes que son profesionales y técnicos, vendedores y trabajadores autónomos con respecto a los nacidos en CataIuña. Pero, examinado más de cerca el fenómeno, llegamos a otra constatación de suma importancia, si nos referimos, por ejemplo, a los profesionales y técnicos que son los sectores de ocupación en los que la educación más podría incidir en la igualdad de oportunidades. Confrontando movilidad con adscripción subjetiva de clase, C. Solé constata que el acercamiento entre inmigrantes y nacidos en Cataluña se da sensiblemente más entre quienes se confiesan de clase baja y mucho menos entre los que se creen de clase media-alta. A pesar de que la autora no va más allá de esta constatación, yo creo que tales datos nos abren perspectivas interesantes, como son: que probablemente entre los técnicos medios-altos se siguen manteniendo las diferencias que ya señalaba Pinilla y que no basta la educación formal para obtener la igualdad de oportunidades, sino que las posibilidades vinculadas al origen social siguen pesando por encima de aquélla.

Pero volvamos al tema de la lengua. Una mayoría de los encuestados para el estudio que comento - $57 \%$ de los nacidos en Cataluña y $60 \%$ de los nacidos fuera- opinan que sabiendo catalán es más fácil encontrar trabajo o cambiar de empleo en Cataluña, al tiempo que casi el $70 \%$ de los inmigrantes asegura que en su lugar de trabajo no necesita saber catalán.

Lo que estas respuestas reflejan -y que posiblemente habría que completar con análisis más objetivos de la estructura categorial de las empresas en relación con el origen geográfico y el conocimiento de la lengua- es que el conocimiento del catalán abre más posibilidades de empleo, o de mejor empleo, para cierto tipo de trabajos -no manuales, servicios cualificados - al tiempo que en los manuales la lengua cuenta poco, puesto que no incide para nada en la productividad (directa o indirecta) del trabajo.

Así, pues, la educación, la lengua y el origen social, aunque con intensidades diferenciadas, son los factores que posibilitan la inserción en la estructura ocupacional a niveles más altos o más bajos, o con opción a movilidad.

A conclusiones semejantes se llega en otro estudio sobre las condiciones de trabajo de la mujer en el Área Metropolitana de Barcelona, ${ }^{7}$ donde se constata que el nivel de cualificación es el ptincipal factor concreto de la discriminación de la mujer tanto en el orden salarial como en las posibilida-

7. F. Miguélez Lobo i equipo: Las condiciones de trabajo de la mujer en Cataluña. Ponencia presentada en el Primer Congreso de Sociología, Zaragoza, septiembre 1981 . 
des de promoción, estableciéndose en la cualificación - y en el nivel de estudios de base- la principal diferencia entre inmigrantes y nacidas en $\mathrm{Ca}$ taluña.

Una primera conclusión a la que podemos llegar es que en el proceso de integración socio-cultural de los inmigrantes éstos están en posiciones bajas en la estructura ocupacional y en ghettos aislados en lo referente al hábitat. Mayores posibilidades educativas dan una cierta movilidad en la segunda generación, en la que, asimismo, se registra un tipo de socialización más congruente con los valores y puntos de teferencia predominantes en esta sociedad.

Pero, en realidad, queda manifiesto lo problemático que resulta hablar de integración a una sociedad urbano-industrial en abstracto. Por encima de la educación y de la lengua, de ser de la primera o de la segunda generación inmigrante, siguen funcionando mecanismos ligados al origen de clase que determinan posibilidades diferenciadas para los individuos en la estructura ocupacional. Es decir, la integración se verifica junto con discriminación, porque la sociedad receptora es una sociedad clasista.

Ello no obsta para que una política educativa dirigida a poner las máximas posibilidades formativas al servicio de los inmigrantes, también por lo que se refiere a la lengua, pueda ser el vehículo de la igualdad de oportunidades que se puede alcanzar en esta sociedad.

\section{CLASE, POLITICA, NACION}

Pero la integración socio-cultural va mucho más allá de la inserción en el trabajo y el hábitat de la sociedad urbano-industrial.

La respuesta a la pregunta sobre identificación de los inmigrantes con Cataluña como realidad nacional es compleja. Por un lado esa identificación supone la aceptación de instituciones sociales y políticas catalanas, y aun de la historia de Cataluña, la adopción de costumbres, valores, pautas culturales y, finalmente, la lengua de la sociedad receptora como propios. Sin embargo, estos procesos, que implican el abandono de la idea de retorno, van imbricados con otros más diferenciados. Me refiero a la diversa vivencia de las instituciones sociales y políticas, y aun culturales, según los propios intereses y las propias condiciones de trabajo y de vida. $\mathrm{O}$, dicho con otras palabras, conciencia nacional y conciencia de clase suponen procesos de integración diferenciables, con lecturas diferenciadas de la realidad social y proyectos políticos parcialmente coincidentes y parcialmente diversos. 
Por ello, y éste es el segundo problema, los estudios sobre actitudes y opciones individuales, aunque necesarios, no bastan. $Y$ con ello quiero señalar una virtud y al mismo tiempo una limitación del estudio de C. Solé frente a quienes quieran elevarlo a conclusiones definitivas de uno u otro signo. En el estudio se señalan una serie de indicadores de identificación con la realidad nacional de Cataluña: la aceptación de la bandera catalana, la participación en la Diada Nacional (11 de septiembre), la aceptación de las instituciones políticas catalanas y de la lengua, la preferencia por organizaciones de clase o nacionalistas. Las conclusiones son que los inmigrantes tienden a considerarse menos nacionalistas que los autóctonos, a preferir menos la «senyera» y participar menos en la Diada, a tener menos interiorizadas las instituciones catalanas. Hasta un cierto punto esto es lo que se puede esperar, puesto que, sencillamente, señala una diferente socialización anterior a la llegada a Cataluña, o una falta de socialización específica en Cataluña, como la propia $C$. Solé hace entrever al señalar que los inmigrantes que llevan más tiempo en Cataluña tienden a identificarse más.

Pero el problema se nos revela más complejo cuando, dando un paso más, ponemos estas actitudes individuales en confrontación con los proyectos socio-políticos de partidos y sindicatos. Los inmigrantes se decantan políticamente en mayor medida por partidos con programas teóricamente menos nacionalistas, aunque de izquierda (PSC y PSUC) que por los partidos llamados nacionalistas (CDC y ERC). Ante una pregunta sobre motivaciones de voto, los inmigrantes señalan que votan a determinado partido porque defiende sus intereses como trabajadores, al tiempo que los autóctonos, sean obretos o sean capas intermedias, exigen que el partido votado sea, antes que nada, nacional catalán. Ello parecería marcar la frontera entre nación y clase en razón de ideologías, visión que parece caer por tierra ante otra correlación en el libro de $\mathrm{C}$. Solé, de la que se deduce que dentro de la misma clase social las diferencias de voto entre autóctonos e inmigrantes son mínimas, cuestión ésta que me parece de suma importancia.

La identificación política por parte de los inmigrantes con la nueva realidad presupone el uso de todos los derechos sociales y políticos, principalmente el de decidir el propio futuro, individual y colectivamente, y el de tener acceso a todas las posibilidades que ofrece esa sociedad, comenzando por el aprendizaje y el uso de la lengua. Ahora bien, tal uso en una sociedad como la catalana está determinado por intereses sociales concretos, por las condiciones de trabajo y de vida, por las solidaridades y los conflictos entre las clases. Es en tal sentido que la investigación que comento ha de ser continuada, con el fin de estudiar la interrelación de los procesos de identificación de clase y de identificación nacional. La investigación de C. Solé señala ya dos elementos de extraordinaria importancia 
a este respecto. Por un lado detecta cómo entre los inmigrantes se da una menor grado de participación en entidades y asociaciones. Por otro, señala que los votantes inmigrantes de algunos partidos de izquierda -es el caso del PSUC - manifiestan un mayor grado de identificación nacional. Ello abre campos interesantes de reflexión, tanto por lo que se refiere a las posibilidades de participación real de los inmigrantes en la vida social, política y cultural como al grado en que las organizaciones políticas y sindicales obreras asumen el problema nacional como algo propio, juntamente con la defensa de los intereses de clase.

El tema sobrepasa los límites y objetivos de este breve artículo, pero la reflexión sobre los programas, la práctica concreta y la organización a nivel nacional (en este caso catalán) de partidos y sindicatos es fundamental para entender las posibilidades reales de integración socio-cultural de los inmigrantes, a menos que se piense que dicha integración es algo meramente individual. Difícilmente, por señalar un aspecto muy concreto, se podrá construir un estado de autonomías, asumido y creído por los ciudadanos, si no existen unas organizaciones sociales y políticas que a nivel de nacionalidad encarnen dicho proyecto. El autocentralismo organizativopolítico de la izquierda en este país no sólo está dejando libre el terreno a las burguesías nacionales, sino que amenaza con vaciar de otra credibilidad que no sea la administrativa los proyectos políticos -que debería querer decir de participación política- de las autonomías. 